\title{
Les mutations des systèmes productifs en France : le cas des filières laitières bovines
}

Mutations of productive systems in France: The case of bovine dairy channels

Daniel Ricard

\section{(2) OpenEdition \\ Journals}

Electronic version

URL: http://journals.openedition.org/rge/5180

ISSN: 2108-6478

Publisher

Association des géographes de l'Est

Printed version

Date of publication: 15 October 2014

ISSN: 0035-3213

\section{Electronic reference}

Daniel Ricard, «Les mutations des systèmes productifs en France : le cas des filières laitières bovines », Revue Géographique de l'Est [Online], vol. 54 / 1-2 | 2014, Online since 22 October 2014 connection on 08 September 2020. URL : http://journals.openedition.org/rge/5180

This text was automatically generated on 8 September 2020

Tous droits réservés 


\title{
Les mutations des systèmes productifs en France : le cas des filières laitières bovines
}

\author{
Mutations of productive systems in France: The case of bovine dairy channels
}

\author{
Daniel Ricard
}

1 Les systèmes productifs français connaissent de considérables mutations qui s'inscrivent en fait dans la lignée d'un mouvement général de progrès que l'on peut faire remonter aux lendemains de la dernière guerre, voire à la révolution industrielle. C'est l'ensemble du et des système/s productif/s qui est/sont concerné/s, en liaison avec le progrès technique (qui améliore la productivité) et les exigences de compétitivité associées à l'élargissement des marchés. L'analyse de ces processus intéresse les géographes comme les économistes, ainsi que d'autres spécialistes des sciences sociales tels les sociologues et peut être conduite globalement (l'économie dans son ensemble) comme à l'échelle d'un secteur d'activité précis : la sidérurgie, la production céréalière, le textile...

2 Notre propos concerne ici les filières agroalimentaires, abordant ainsi deux systèmes productifs : l'agriculture et l'industrie, agroalimentaire en l'occurrence. On rejoint là un positionnement classique, les géographes ruralistes ayant l'habitude de raisonner en filière, dans la lignée des travaux de J. Renard, C. Canévet, J.-P. Charvet, G. Dorel, J. Vaudois ou J.-P. Diry pour n'en citer que quelques-uns.

Il y a autant de filières agroalimentaires que de productions agricoles, mais certaines se sont affirmées plus que d'autres, en lien avec leur dynamisme et surtout leur envergure économique. S'individualisent alors des filières céréalières, sucrières ou laitières, d'autres structurées autour des fruits et légumes ou des produits carnés (viande de boeuf, de porc, de volaille...), sans oublier les filières viticoles, championnes de la création de richesse. Chacune a ses spécificités, mais toutes comportent une dimension géographique déterminante. 
4 Le sujet incite à conduire l'analyse, en première approche, à l'échelle nationale, mais ces filières s'inscrivent aussi dans un cadre européen. L'agriculture est en effet gérée depuis 1962 par une Politique Agricole Commune (PAC) omniprésente, les différentes Organisations Communes de Marché (OCM céréales, lait, viande bovine, sucre...) influençant aussi le secteur de la transformation. En outre, la dimension mondiale du secteur agricole et des filières n'est jamais loin. D'un côté, le commerce international est ancien ${ }^{1}$ mais de l'autre, l'élargissement progressif des échanges est assez récent. Les filières agroalimentaires ont aussi une dimension régionale, voire locale importante, avec des caractéristiques et des évolutions souvent fort différenciées sur le plan spatial, de telles différenciations ne renvoyant pas seulement aux classiques oppositions plaine / montagne, privé / coopératives ou grande industrie / artisanat.

5 Le monde laitier mérite une attention particulière à plus d'un titre. Il s'agit tout d'abord d'un exemple caractéristique de métier structurellement organisé en filière: 98,2\% de la production de lait en France est en effet collectée (CNIEL $\left.{ }^{2}, 2012\right)$, donc destinée à une transformation en laiterie, ne laissant que des miettes à la transformation fermière (reblochon, saint nectaire...) ou à des circuits courts dont on voit qu'il faut en relativiser l'ampleur. Il y aura donc des interrelations très fortes, quasi obligatoires, entre l'amont et l'aval.

6 Seconde caractéristique : l'ampleur de cette filière. La France est le second pays laitier européen derrière l'Allemagne et la profession fait preuve d'une réelle l'efficacité, en liaison avec la performance des élevages et des entreprises de transformation et un héritage incomparable de savoir faire, notamment fromagers, le tout stimulé par des habitudes de consommation qui font une large place aux produits laitiers.

7 Troisième point, cette performance d'ensemble de la filière nationale se réalise sur un fond de mutations considérables, mutations qui animent autant l'amont (les exploitations) que l'aval (les laiteries), qui sont à la fois techniques et économiques et qui tendent à s'accélérer dans la période récente. Ce sera le cœur de notre propos.

\section{Les filières laitières françaises : un monde de complexité}

8 Le lait en France peut se définir par quelques chiffres: une production de 24,34 milliards de litres, une collecte de 23,91 milliards fournie par 72328 producteurs élevant 3660300 vaches laitières, des livraisons moyennes par exploitation de 330 600 litres par an (données CNIEL, 2011), 1290 entreprises de transformation employant 56465 salariés et un chiffre d'affaires de 25 milliards d'euro... soit autant que l'industrie pharmaceutique (INSEE)!

9 Ces chiffres cachent en fait une très grande diversité et des intervenants fort différents : producteurs, collecteurs, transformateurs, affineurs parfois, commerciaux et distributeurs, sans oublier en amont, marchands d'aliments et de matériel, inséminateurs ou vétérinaires, voire, plus en amont encore, chercheurs et techniciens. On rejoint là la notion de filière, avec ses maillons interdépendants. Ceux de la production et de la transformation sont à l'évidence les pivots du système. 


\section{A. À l'amont : des producteurs et des mécanismes de concentration} même si certaines régions ont toujours été plus spécialisées que d'autres. Pr même si certaines régions ont toujours été plus spécialisées que d'autres. Puis la profession a connu une intense concentration selon des processus qui influencent d'ailleurs à la fois les effectifs et la géographie de la production.

11 Ce mécanisme de restructuration remonte en fait au début des années $1960^{3}$. Structurel, il renvoie à la spécialisation progressive des fermes, mais connaît des accélérations dans la période récente. Dans les années 1970, il est stimulé par la mécanisation de la traite qui marginalise ceux qui refusent de s'adapter ${ }^{4}$. La restructuration s'accélère avec l'instauration des quotas en 1984. Ce blocage des livraisons impacte surtout les petits livreurs, rarement considérés comme prioritaires pour bénéficier de quotas supplémentaires. L'écrémage est alors sévère puisque l'on passe de 384945 livreurs de lait en 1983 à moins de 200000 dès 1991. La restructuration s'accélère à nouveau depuis dix ans, la baisse de la rentabilité au litre obligeant les éleveurs à produire plus pour compenser la hausse des coûts de production alors que le prix du lait stagne. Les livreurs sont moins de 100000 dès 2005 puis 72328 en 2011. En 40 ans, les effectifs ont donc été divisés par dix, pour une production grossièrement identique : on mesure les progrès réalisés en matière de productivité !

Fig. 1 : Les structures de production laitière en France

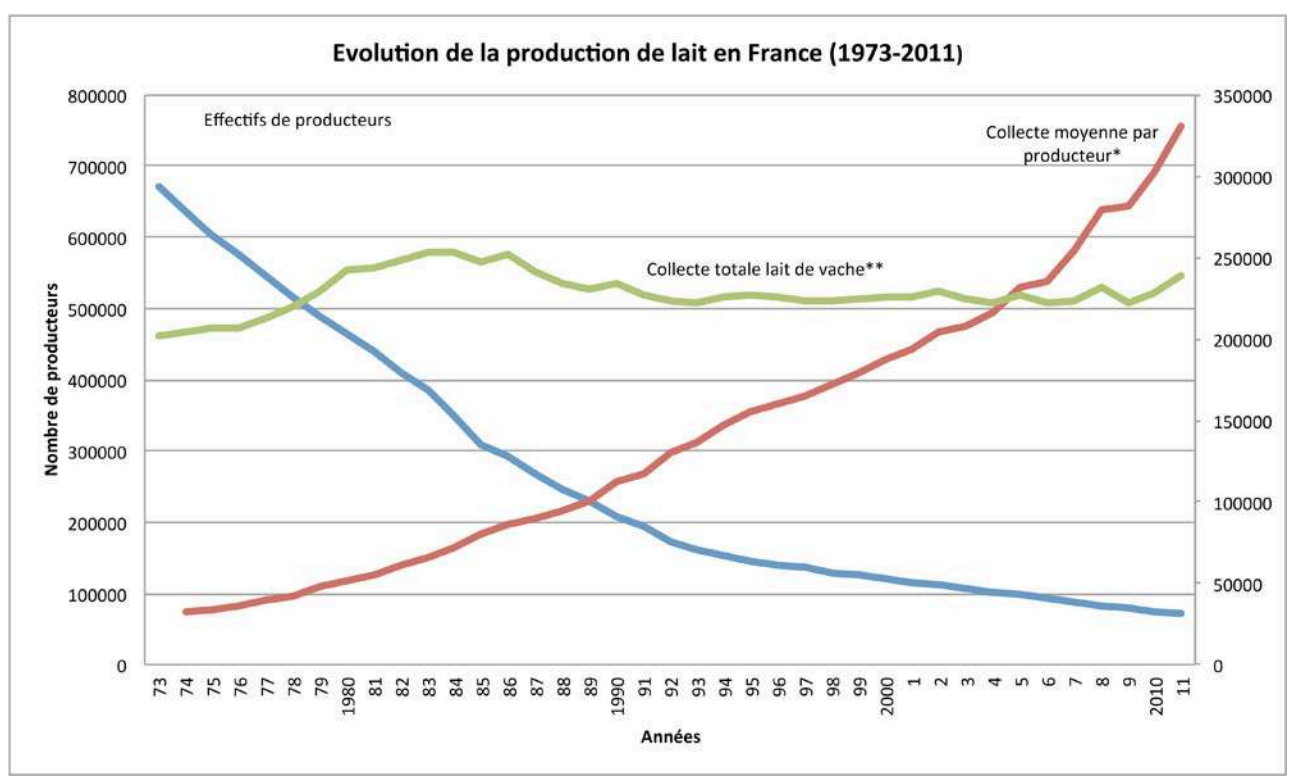

* Collecte moyenne en litres par an, échelle de droite ; ** Collecte totale en 100000 litres, échelle de droite (200 $000=20$ milliards de litres).

Source : CNIEL

12 L'amont de la filière se caractérise aussi par une géographie de la production qui montre un certain nombre de spécificités. Le premier ensemble régional est constitué par le «Grand Ouest » qui assure 48,1\% de la collecte nationale (CNIEL, 2011). Ces plaines océaniques associent toutefois des territoires à la genèse très différente. La Basse Normandie est une vieille région laitière puisant ses racines dans la tradition fromagère locale et l'action des fabricants de camembert, sans oublier la réputation des beurres et crèmes (d'Isigny). La Bretagne produisait surtout du beurre fermier, exporté 
certes jusqu'en Angleterre, mais dans le cadre d'une agriculture médiocre. La révolution agricole bouleverse la donne, ici comme dans les Pays de la Loire (Canévet, 1991 et 1992). La nouvelle dynamique impulsée entre autres par la Jeunesse Agricole Chrétienne (JAC) rencontre alors, dans les années 1960, l'action stimulante des entreprises de collecte, coopératives ou privées, pour constituer très vite l'un des premiers bassins laitiers européens! Le mouvement se fonde sur l'intensification de la production et l'industrialisation de l'aval et touche aussi la Normandie.

Toute une couronne laitière court ensuite de la vallée de la Seine à l'Alsace, longeant les frontières en contournant le Bassin parisien céréalier. Elle assure $20 \%$ de la collecte nationale, certains secteurs constituant d'ailleurs de gros bassins laitiers : Caux, Pays de Bray, Boulonnais, Thiérache, Ardennes, Meuse, Ouest vosgien. Toutes ces plaines au climat favorable à l'herbe font cohabiter des régions de tradition laitière (Bray, Caux, Thiérache...) et d'autres qui se sont spécialisées plus tard, sous l'impulsion des industriels (Nord et Pas-de-Calais ${ }^{5}$ ) ou des coopératives (Meuse).

La dimension montagnarde de la production est importante puisqu'elle concerne environ $12 \%$ de la collecte nationale. La montagne est structurellement à la peine sur le plan technique ${ }^{6}$ mais elle abrite un important patrimoine de savoir faire fromagers à l'origine de noyaux productifs significatifs en Savoie, dans le Jura et le Massif central. On oublie souvent que la montagne a aussi connu la révolution agricole qui a permis l'émergence de nouvelles régions laitières, souvent très dynamiques, à partir des années 1960/70 : Ségalas de l'Aveyron, Châtaigneraie cantalienne, Monts du Lyonnais et du Forez, Pilat.

Fig. 2 : La localisation de la production laitière en France (2012)

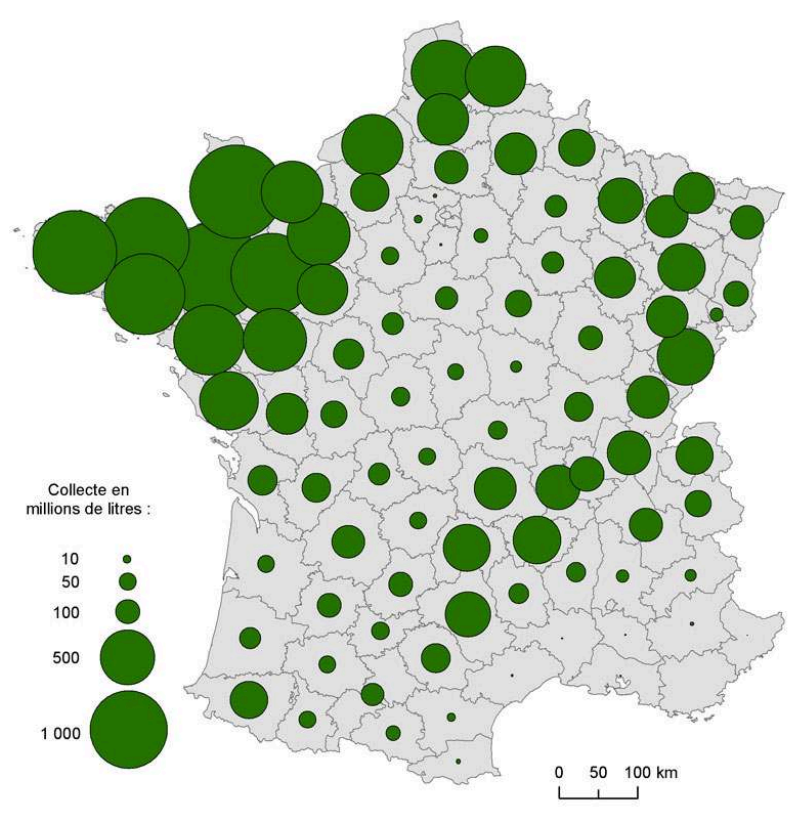

Le reste du pays ressemble, en revanche, à des périphéries laitières, avec des producteurs rares et des volumes faibles et souvent en recul' ${ }^{7}$. C'est le cas des plaines 
sédimentaires où la concurrence des céréales est forte, même si Poitou-Charentes conserve encore quelques volumes de lait. La restructuration, très intense, y a permis l'émergence de quelques très gros producteurs intensifss. Autre périphérie en crise, le monde méditerranéen. La production s'y réfugie en altitude mais, dans les HautesAlpes comme en Cerdagne, la collecte est structurellement en déclin.

\section{B. L'aval de la filière}

La transformation, également soumise à un puissant mouvement de concentration (voir plus loin), se caractérise par une grande diversité des acteurs, plus marquée que dans le Nord du continent où la grande industrie domine tout. Cette diversité renvoie à l'histoire.

Jusqu'en 1945, on comptait une multitude de transformateurs fermiers et d'artisans, surtout fromagers, avec beaucoup de coopératives: fruitières franc-comtoises et savoyardes, coopératives de Poitou-Charentes, du Cantal, de Bresse ou de la Dombes. Les fromagers privés étaient également nombreux, surtout dans le monde des pâtes molles (brie, camembert...), un peu moins dans celui des pâtes pressées cuites (gruyères) dominé par la coopération (Delfosse, 2007). Ajoutons enfin de nombreux affineurs, privés, comme dans le Jura, en Savoie et dans le Cantal.

Après guerre, la croissance favorise l'émergence de nouveaux acteurs. La plupart des grandes coopératives actuelles ${ }^{9}$ se développent alors fortement, en investissant beaucoup dans le créneau de l'approvisionnement urbain en lait et en produits frais, comme autour de Paris et de Lyon. C'est typiquement le cas de celles qui seront à l'origine de SODIAAL ${ }^{10}$ après avoir créé les marques Candia et Yoplait. Dans les années 1960-70, la coopération investit aussi dans le couple beurre + poudre ${ }^{11}$, surtout dans le Grand Ouest, stimulée par la politique incitative de Bruxelles. L'Union Laitière Normande (ULN) devient alors la première entreprise laitière française, avant de disparaître en 1992. Bien d'autres coopératives se développement pour vite atteindre une taille régionale, comme l'Union Laitière de la Meuse (qui ne transforme pas), l'ULPAC à Toulouse qui fusionnera en 1988 avec la cantalienne Centre Lait, l'Union Laitière Vittelloise (Vosges), l'Union Agricole Comtoise, la coopérative d'Isigny et les Maîtres Laitiers du Cotentin dans la Manche, la Prospérité Fermière (Nord), Alsace Lait ou encore le Groupement Laitier de l'Association Centrale (Surgères, CharenteMaritime). D'autres grosses unités accompagnent la révolution agricole dans l'Ouest: Even à Ploudaniel, Coopagri Bretagne (issue de l'office Central de Landerneau) et sa concurrente UNICOPA ont toutes leurs racines dans le Nord Finistère, haut lieu du dynamisme breton. Citons enfin la CANA (Ancenis) et les coopératives qui constitueront le groupe nantais Eurial.

19 On assiste aussi à l'expansion de plusieurs grosses sociétés privées très dynamiques, nées de petites affaires locales. Citons alors Bongrain (Haute-Marne), Entremont (Annecy, puis Bretagne), Senoble (Yonne), les Fromageries Bel (aux racines plus anciennes), Bridel (Retiers, Ille-et-Vilaine) et surtout Besnier. Cette petite fromagerie de Laval rebaptisée Lactalis est devenue l'un des leaders mondiaux de la transformation laitière, aux côtés de Danone et du suisse Nestlé. L'histoire de Danone est très différente, puisqu'il s'agit là d'un groupe industriel côté en bourse, constitué au gré d'OPA et de réorientations stratégiques successives. Toutes ces firmes développent des marques fortes (La Vache qui Rit pour Bel dès 1921, Caprice des Dieux dès 1956 pour 
Bongrain, Entremont, Bridel, Senoble, Président pour Besnier en 1968, Danone ou Activia pour Danone...). Elles connaissent une très forte croissance à partir des années 1970, en France puis à l'international, même si certaines disparaissent avec le temps (Bridel racheté par Lactalis en 1990, Entremont repris par SODIAAL en 2011...).

Derrière ces grands groupes, de nombreuses PME prospèrent sur des marchés de niche, comme Triballat (Cher) avec sa marque Rians et ses fromages de chèvre. Le secteur des fromages d'appellation d'origine protégée (AOP) s'individualise assez nettement grâce à des produits à bonne valeur ajoutée qui ont permis de conserver, au moins dans le Jura et en Savoie, un dense tissu de fruitières villageoises et des affineurs réputés tels Arnaud (marque Juraflore) à Poligny, la capitale du comté.

\section{Les principaux facteurs de la mutation des filières}

Depuis 50 ans, la filière laitière française connaît de profondes transformations. Les facteurs de cette mutation sont nombreux, liés à la marche générale de l'économie ou, plus spécifiquement, à l'environnement laitier. Ceux qui relèvent de la politique agricole et des forces du marché mériteront toute notre attention.

\section{A. Le poids de la politique agricole commune}

La PAC est en effet un élément clef, à l'amont comme à l'aval, en liaison avec une OCM lait évoluant elle-même au gré des réorientations successives de cette PAC.

En 1962, Bruxelles instaura des prix d'intervention ${ }^{12}$ élevés et un système douanier très protecteur ${ }^{13}$ qui stimulèrent la production. Dès les années 1970, celle-ci dépasse la capacité du marché intérieur alors que se développe la fabrication de beurre et de poudre de lait, invendables certes mais stockés par l'Europe. Bretagne et Pays de la Loire sont en pointe dans cette dynamique (Canévet, 1992), jusqu'à ce que la surproduction ne devienne insupportable.

Cette impasse conduit Bruxelles à instaurer, en 1984, des quotas. La collecte, contingentée, est réduite de 12\% environ jusqu'en 1992 et l'on s'attaque aux avantages de l'intervention. Les stocks se dégonflent alors progressivement à partir de la fin de la décennie ${ }^{14}$, assainissant ainsi le marché. Ces quotas ont une influence structurelle sur la filière. A l'amont, seuls les livreurs jugés prioritaires (jeunes, investisseurs...) bénéficient de droits à produire supplémentaires tandis que des dizaines de milliers d'autres, souvent petits et/ou âgés, préfèrent les aides à la cessation laitière, souvent généreuses. A l'aval, la baisse de la collecte rompt avec la croissance vigoureuse des années précédentes, stimulant la restructuration des sites industriels et des entreprises.

A la fin du siècle, la réflexion progresse à propos d'un abandon des quotas. Elle souligne des positionnements différents, libéraux (Europe du Nord) ou plus interventionnistes (Europe du Sud, Autriche...), la France penchant plutôt dans le second camp, le tout sur fond d'élargissement de l'Union (à des pays finalement peu laitiers, sauf la Pologne, mais appelés à être gourmands en fonds communautaires). Cette réflexion débouche sur les Accords de Luxembourg (2003) qui constituent une rupture importante. Les quotas sont conservés mais l'intervention est réduite en volume et les prix indicatifs diminués, compensés par une aide forfaitaire découplée de 35,5 euro/tonne de lait, 
intégrée aux DPU ${ }^{15}$ des agriculteurs. Ceux-ci ne bénéficient plus que d'un prix minimal garanti voisin de 210 euro/tonne, largement insuffisant, alors qu'à l'aval les fabricants de beurre et de poudre ne peuvent plus, eux non plus, vivre de l'intervention. Bref, il ne s'agit plus que d'un simple filet de sécurité, réservé aux situations de crise grave. La filière est davantage confrontée au marché et le prix du lait devient plus volatil ${ }^{16}$, une vraie rupture pour les producteurs. La qualité des débouchés commerciaux devient décisive pour des transformateurs habitués jusque là à plus de lisibilité, un mauvais positionnement pouvant leur être fatal. La concentration s'accélère.

Avril 2015 verra la suppression des quotas, une étape moins redoutée qu'il y a quelques années car le contexte s'est renouvelé. D'une part, une contractualisation se met en place entre producteurs et laiteries, devant permettre à la filière de gérer les volumes, au niveau des entreprises cette fois. Mais surtout, les marchés internationaux semblent davantage porteurs, et ce de manière structurelle. Selon la FAO, la consommation mondiale de lait augmenterait de 2,3\% par an jusqu'en 2023 contre 1,8\% seulement pour la production. Ce déséquilibre est perçu comme un atout, d'autant plus que les écarts de compétitivité entre les grands exportateurs de lait (Europe, Etats-Unis, Océanie) diminuent beaucoup depuis dix ans.

Ce nouvel environnement est propice à des évolutions différenciées sur le plan géographique. A l'échelle de l'Europe, les régions et les entreprises les mieux placées sont l'Allemagne du Nord, nouveaux länder compris, le Danemark et la Scanie, les PaysBas (avec de sérieuses limites environnementales) et l'Irlande (avec un système herbager moins intensif). En France, le Grand Ouest semble le plus concurrentiel quand d'autres régions paraissent condamnées (Provence, zones céréalières, montagnes aux filières mal structurées...).

\section{B. Le rôle du marché}

Pour les filières, le rôle des consommateurs est bien entendu décisif, même si le marché n'est pas totalement libéralisé. Au sein de l'Union Européenne, les échanges sont libres mais l'OCM lait oriente significativement le fonctionnement du marché et, à un autre niveau, les produits agricoles n'ont intégré les mécanismes de l'OMC qu'en 1992 (Uruguay round). Bref, les échanges agricole et agroalimentaire mondiaux restent encadrés, même si l'on tend vers une libéralisation évidente de ceux-ci.

Au-delà des politiques agricoles, l'attitude des consommateurs est un facteur évolutif essentiel pour les filières laitières, avec des mutations qui renvoient à l'ouverture progressive des frontières et au développement des échanges, à la dynamique commerciale des entreprises et aux habitudes de consommation dont la dimension culturelle est toujours importante. Les Grecs se nourrissent de feta, Irlandais et Finlandais boivent énormément de lait... Les Français eux consomment beaucoup de fromage, au point d'en faire un plat à part entière dans l'ordonnancement du repas (Fumey, Etcheverria, 2004). Le pays abrite d'ailleurs nombre de fromages de terroir, progressivement protégés par des AOP. Ces habitudes de consommation peuvent être régionalisées, le beurre cédant ainsi le pas devant l'huile d'olive en milieu méditerranéen. Notons aussi que les migrations font évoluer cette géographie, Paris étant par exemple devenu un pôle de consommation des fromages d'Auvergne grâce aux migrants venus du Massif central. 
30 A partir d'une situation "traditionnelle ", héritée de l'histoire et marquée par la consommation de produits «classiques" (fromages de terroir, beurre, crème), les années 1970/80 voient l'émergence de nouveaux produits qui constituent autant de relais de croissance pour les entreprises, stimulés par la généralisation de la grande distribution et de la chaîne du froid ainsi que par les nouvelles méthodes de marketing. Dès lors, la consommation de yaourts, de desserts lactés, de crème, de glace et de fromages à marques commerciales (Président, RichesMonts, Entremont, Caprice des Dieux, Tartare, Chaume...) progressent considérablement. A l'inverse, la consommation de beurre et de lait (UHT) diminue structurellement.

Dans le même temps, l'internationalisation des échanges s'affirme et, si la dimension nationale de la consommation demeure, les produits se diffusent au-delà des frontières. D'un côté, lait UHT ou fromages ingrédients ${ }^{17}$ (pour les pizzas, la restauration rapide...) pénètrent en France, mais les entreprises nationales, très dynamiques, exportent massivement, en Europe mais aussi, et de plus en plus, sur des marchés lointains à la croissance spectaculaire : Chine, Russie, Brésil, Asie du Sud-est... Bref, le marché joue à plein et l'internationalisation des échanges est évidente. En 2012, la France importe l'équivalent de 5,2 milliards de litres mais en exporte 10,2 milliards, soit $42,5 \%$ de la collecte nationale! Les exportations atteignent 6,63 milliards d'euro, dont 4,55 vers l'Europe et 2,08 vers les pays tiers (contre seulement 1,14 milliards en 2002). A la sortie, la filière dégage un surplus commercial de 3,5 milliards (Revue Laitière Française $n^{\circ} 728$, janvier 2013).

\section{La mutation des filières laitières}

Il convient de bien différencier les facteurs de la mutation (les éléments qui stimulent le changement) des mutations elles-mêmes, sachant que l'ensemble est souvent lié. Ainsi, pour les yaourts et les desserts lactés, la mutation vient-elle de la demande des consommateurs ou bien de la créativité des fabricants? On doit aussi distinguer les évolutions structurelles de long terme (la révolution agricole, l'industrialisation...) des changements contemporains et plus spécifiques à la filière étudiée. Nous nous concentrerons bien entendu sur ces dernières, bien qu'elles doivent être replacées dans un contexte plus large.

\section{A. À l'amont, un monde de la production en profonde mutation}

Le processus déjà évoqué de concentration accélérée des producteurs fait partie d'une mutation contemporaine des filières qui comporte aussi une double dimension technique et géographique.

\section{Sur le plan technique}

La production laitière vit, depuis $50 / 60$ ans, de considérables évolutions qui bouleversent le métier. On rejoint là la révolution agricole qui a permis le passage à une production modernisée, sur fond de spécialisation des fermes.

Jusque dans les années 1950/60, cette production est héritée de l'histoire, fondée sur des races locales, le pâturage et le foin. Quelques régions apparaissent en avance et mieux spécialisées, comme la Normandie, mais aussi plusieurs régions d'altitude 
comme le Jura qui s'appuie sur une race Montbéliarde précocement sélectionnée. Enfin, dans certaines banlieues, le marché local, captif et en croissance, stimule une production à l'auge très intensive : Paris, Marseille, la Riviera...

La révolution agricole généralise un nouveau modèle associant vache Holstein ${ }^{18}$, maïs fourrage et ensilage. Cette ration de base, très énergétique, est ensuite complémentée par des aliments pour le bétail à base de céréales et de soja qui font augmenter la production, dès lors que l'animal en a le potentiel génétique. Ce modèle, qui associe aussi insémination artificielle et mécanisation de la traite, s'exprime le mieux et le plus tôt en plaine, comme dans le Nord-Pas-de-Calais et surtout en Bretagne / Pays de la Loire, à partir du milieu des années 1960. Il se diffuse ensuite dans le reste du pays, y compris dans des petites Bretagne montagnardes (Châtaigneraie cantalienne dès 1961, Monts du Lyonnais, Pilat, Ségalas...). D'autres massifs, qui doivent souvent remplacer le maïs par l'herbe, adoptent ce modèle alors que les quotas viennent d'être instaurés, ce qui rend leur situation délicate (Margeride, Champsaur, Devès...). Les régions qui restent à l'écart de cette dynamique sont bien individualisées, comme le Jura et les Alpes du Nord qui, par volonté locale de respect du terroir, refusent l'ensilage et privilégient les races locales. L'expérience montre qu'elles ont eu raison.

De nos jours, la révolution agricole perdure, parfaitement visible dans la mécanisation de la fenaison, mais trois éléments spécifiques au monde laitier doivent être abordés.

- Depuis 20 à 25 ans, le processus de sélection génétique s'intéresse de plus en plus à la qualité du lait. On privilégie alors la matière protéique (qui sert à fabriquer le fromage), au détriment de la matière grasse ${ }^{19}$, sous la pression des grilles de paiement du lait. Depuis quelques années, l'arrivée de semences sexées bouleverse la sélection génétique en permettant d'avoir plus de femelles, donc d'accélérer le renouvellement du troupeau.

39 - La dimension sanitaire est importante. On la retrouve dans la sélection génétique par élimination de sujets potentiellement peu intéressants mais l'élément clef apparait bien la coûteuse mise aux normes des bâtiments d'élevage (stockage du lisier...) qui, depuis 10 à 15 ans, est un accélérateur de la concentration des élevages.

40 - La réduction de la charge de travail semble l'aspect le plus décisif, les éleveurs recherchant là une amélioration de leurs conditions de vie. La constitution de GAEC permet de libérer des week-ends. On délaisse les étables entravées et la distribution de la nourriture se fait de plus en plus par un couloir d'alimentation frontal, souvent au moyen de mélangeuses distributrices achetées parfois en commun. On tend à distribuer une fois par jour, voire 6 fois par semaine, et non plus avant chaque traite. On cherche enfin à contourner l'astreinte de la traite au moyen d'installations plus performantes (salles de traite à grande capacité, systèmes rotatifs), de robots de traite ${ }^{20}$, voire en supprimant la traite du dimanche soir.

41 La production laitière devient donc plus rationnelle, réalisée dans des ateliers (de plaine) de plus grande taille, avec des animaux qui se contentent souvent d'une aire d'exercice, sont nourris à l'auge (sans que l'on puisse parler de production hors sol) et traits dans des conditions renouvelées. La productivité progresse spectaculairement et les livraisons moyennes par exploitation doublent entre 1998 et 2012 tandis que les petits livreurs sont marginalisés. En 2011, ceux qui disposent de moins de 150000 litres de référence ${ }^{21}$ (soit 25 vaches) ne possèdent que $4,6 \%$ de la référence nationale quand les détenteurs de plus de 400000 litres de quota en contrôlent $51,8 \%$. 


\section{Sur le plan géographique} agriculteurs totalement libres de produire. La collecte connaît alors un développement spectaculaire dans l'Ouest. Elle croît de 1,593 milliards de litres en Bretagne, de 1,34 milliard dans les Pays de la Loire et de 457 millions en Basse-Normandie, les plus fortes augmentations concernant l'Ille-et-Vilaine et les Côtes-d'Armor. Une dynamique à peine moins spectaculaire anime le Nord et le Nord-Est du pays, voire le piémont pyrénéen. Toutes ces plaines propices à l'herbe et au maïs disposent d'un vrai potentiel de développement.

Fig. 3 : L'évolution de la collecte $1976 / 84$ et $1984 / 2012$
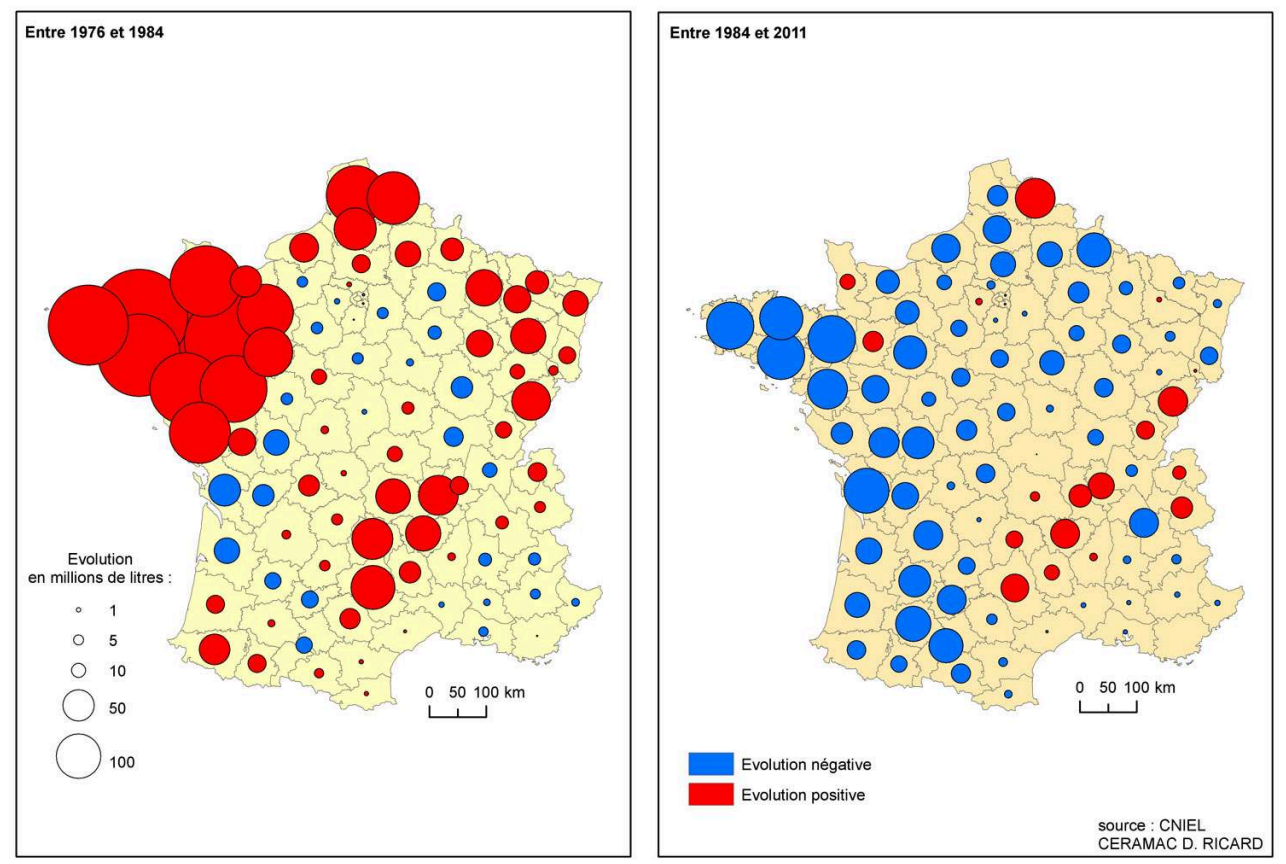

La croissance est également très nette en montagne où le lait apparaît comme une production bien adaptée. Il progresse dans les vieilles régions fromagères (Jura, Savoie, Auvergne) mais aussi du Rhône à l'Aveyron où la dynamique rappelle celle du Grand Ouest.

À l'inverse, le lait recule en milieu méditerranéen (où il était déjà rare) et dans de nombreuses régions de plaine où la concurrence des cultures est intense : cœur du Bassin parisien, Bassin aquitain, Charente-Maritime, Vienne.

Après 1984, la donne change. Les quotas font nettement reculer la collecte mais, de plus, l'administration opère des redistributions des droits à produire entre les territoires. Elle choisit ainsi, dans les années 1980, de privilégier la montagne au moyen de prélèvements significatifs opérés sur la plaine. La collecte évolue donc sous le double effet de la stratégie des agriculteurs et de la politique agricole. 
47 Le Grand Ouest enregistre curieusement le plus fort recul au niveau national, en rupture totale avec la période précédente. La dynamique laitière y reste très présente mais la gestion des quotas y fait nettement reculer la collecte, sauf en Mayenne et dans la Manche. Les autres régions de plaine aussi connaissent souvent une baisse des volumes, mais dans un contexte marqué par la concurrence avec les céréales. Le recul est de l'ordre de $20 \%$ dans le Bassin parisien, de 30 à $40 \%$ dans le Bassin aquitain et en Poitou-Charentes, et même de $56 \%$ dans le Gers, mettant les entreprises locales en difficulté. Le déclin est encore plus prononcé en Provence.

48 Les secteurs en croissance sont rares, avec le Nord et surtout les départements d'altitude qui, outre un traitement favorable de l'administration, ont moins de possibilités de réorientation productive et profitent, dans le Jura et en Savoie, de l'efficacité des filières fromagères.

\section{B. A l'aval, des mutations complexes}

49 L'aval de la filière connaît aussi d'intenses mutations, à l'image de l'ensemble de l'industrie française, des changements qui sont au moins de trois type: techniques, productifs et capitalistiques.

\section{Les mutations techniques}

Les mutations techniques renvoient à la notion de productivité et au processus d'innovation. La recherche d'une meilleure productivité est structurelle dans le monde laitier, conduisant à des usines toujours plus vastes. De nos jours, les plus grosses fromageries fabriquent 50000 tonnes par an, utilisant donc 500 millions de litres de lait: une cinquantaine de semi-remorques par jour (Herbignac en Loire Atlantique, Domfront dans l'Orne...). Pour le lait de consommation, qui ne procure que de très faibles marges, le seuil de rentabilité dépasse cent millions de litres. A la clef, le nombre de sites de production se réduit considérablement. On passe ainsi, de 1981 à 2011, de 1447 fromageries à 534, de 949 beurreries à 166, le nombre de sites de production de lait liquide passant même de 943 à 57 et celui des tours de séchage de 130 à 11 ! Et 15 ateliers suffisent pour assurer $74,8 \%$ de la production nationale de lait, 11 pour $82,8 \%$ de celle de beurre. La concentration est un peu moins nette en fromagerie compte tenu de la présence de nombreux ateliers artisanaux, surtout en AOP, mais les 50 plus gros sites (plus de 10000 tonnes chacun) livrent $74,1 \%$ de la production nationale (CNIEL, 2012).

51 Cette industrialisation se traduit par un recul progressif des emplois, contrebalancé toutefois par de nouveaux besoins liés à la recherche de valeur ajoutée et aux exigences sanitaires et commerciales. Les effectifs salariés passent ainsi de 98670 en 1974 (maximum historique) à 58823 en 1996 avant de se stabiliser depuis (56 465 en 2010, CNIEL). Les plans sociaux concernent finalement assez peu la filière, à la différence, par exemple, de l'actualité récente des abattoirs.

Sur le plan technique, les années 1960 avaient vu la mise au point de l'industrialisation de la fabrication de l'emmental pasteurisé et la diffusion de la technique de l'UHT (chauffage du lait de consommation à Ultra Haute Température, à $140 / 150^{\circ}$ avant empaquetage). L'innovation induit alors une mutation géographique majeure puisque l'emmental breton supplante rapidement les fabrications traditionnelles de l'Est 
Central (Franche-Comté, Savoie) au lait cru qui résistent difficilement en dépit de l'obtention d'un Label Rouge (1979) puis d'une IGP (1996). Le lait UHT, qui se conserve des mois à température ambiante, bouleverse les circuits de distribution en supprimant la nécessaire proximité géographique entre producteurs et consommateurs. Les villes méditerranéennes peuvent alors s'approvisionner auprès d'usines et de zones laitières éloignées mais performantes (Vienne, Toulouse, pourquoi pas le Grand Ouest ou l'Allemagne), ce qui contribue au déclin des bassins laitiers proches insuffisamment performants : Cerdagne, Vaucluse, basse vallée du Var, Alpes au sud de la Durance). La microfiltration, mise au point dans les années 1980 et qui permet de retenir les bactéries indésirables par des membranes filtrantes, améliore la sécurité sanitaire, accroit les rendements laitiers (cas de la Fromagerie Guilloteau qui initie cette pratique dans le Pilat avec son Pavé d'Affinois), préserve les qualités organoleptiques et allonge le délai de consommation du lait frais ${ }^{22}$. Depuis 20 ans, d'autres innovations technologiques permettent d'extraire de nouvelles molécules dans les poudres (lactose, lactoprotéines...) ou bien par cracking de la matière grasse, ce qui offre au secteur de nouvelles opportunités commerciales. Ainsi, la poudre de lactosérum, jusque là destinée à l'alimentation pour le bétail, trouve de nouveaux débouchés, surtout en Asie, et pour l'alimentation humaine. La mutation technique n'ignore pas les artisans, à l'image de la filière comté qui a largement modifié ses équipements et équipé ses caves de robots.

\section{Les mutations productives}

La mutation technique, celle des habitudes alimentaires et les nouvelles opportunités à l'exportation conduisent à des évolutions différenciées des types de fabrications, le tout à partir d'une ressource stabilisée en volumes depuis 30 ans.

Le secteur le plus dynamisme concerne les produits frais : yaourts, desserts lactés et crèmes de consommation, dont les fabrications sont respectivement multipliées par 3,$9 ; 6,3$ et 5,6 de 1975 à 2011 (CNIEL)! La demande croissante des consommateurs, la praticité de ces produits et l'efficacité d'entreprises aux marques réputées (Senoble, Yoplait, Calin, La Laitière, Mamie Nova, Activia, Danette, Danone...) expliquent ce succès. Ces produits sont essentiellement vendus en Europe, mais les groupes diffusent aussi ces marques à l'étranger, au moyen de franchises (Yoplait) ou en produisant sur place.

La production fromagère, elle aussi en forte croissance (x 1,93), s'appuie sur une importante tradition, un gros patrimoine de savoir faire ${ }^{23}$ et, encore une fois, l'efficacité des entreprises. Elle est capitale pour la filière car elle utilise $36,8 \%$ du lait national contre $7 \%$ seulement pour les yaourts et desserts, $10,6 \%$ pour le lait liquide, $13,8 \%$ pour les poudres et $18,7 \%$ pour le beurre (CNIEL, 2012). La France, longtemps leader européen, a été dépassée par l'Allemagne en 2003, un pays davantage spécialisé dans les fromages ingrédients. Elle mise davantage sur les produits de terroir et surtout sur les spécialités à marque commerciale (RichesMonts, Entremont, Président, Lepetit, Paysan Breton, Lescure, Caprice des Dieux, Chaumes, Cour de Lion...).

Le lait de consommation, hier en forte croissance, culmine en 1994 avant de devenir un marché mature, difficile pour des entreprises confrontées à la baisse des achats, à la pression de la grande distribution qui en a fait un produit d'appel et désormais aux importations d'Europe du Nord (environ $12 \%$ du marché). La restructuration fait donc 
rage, illustrée par la fermeture en cours des trois usines SODIAAL de Vichy, du Ludre et de Villefranche-sur-Saône.

Fig. 4 : L'évolution des principales fabrications laitières en France
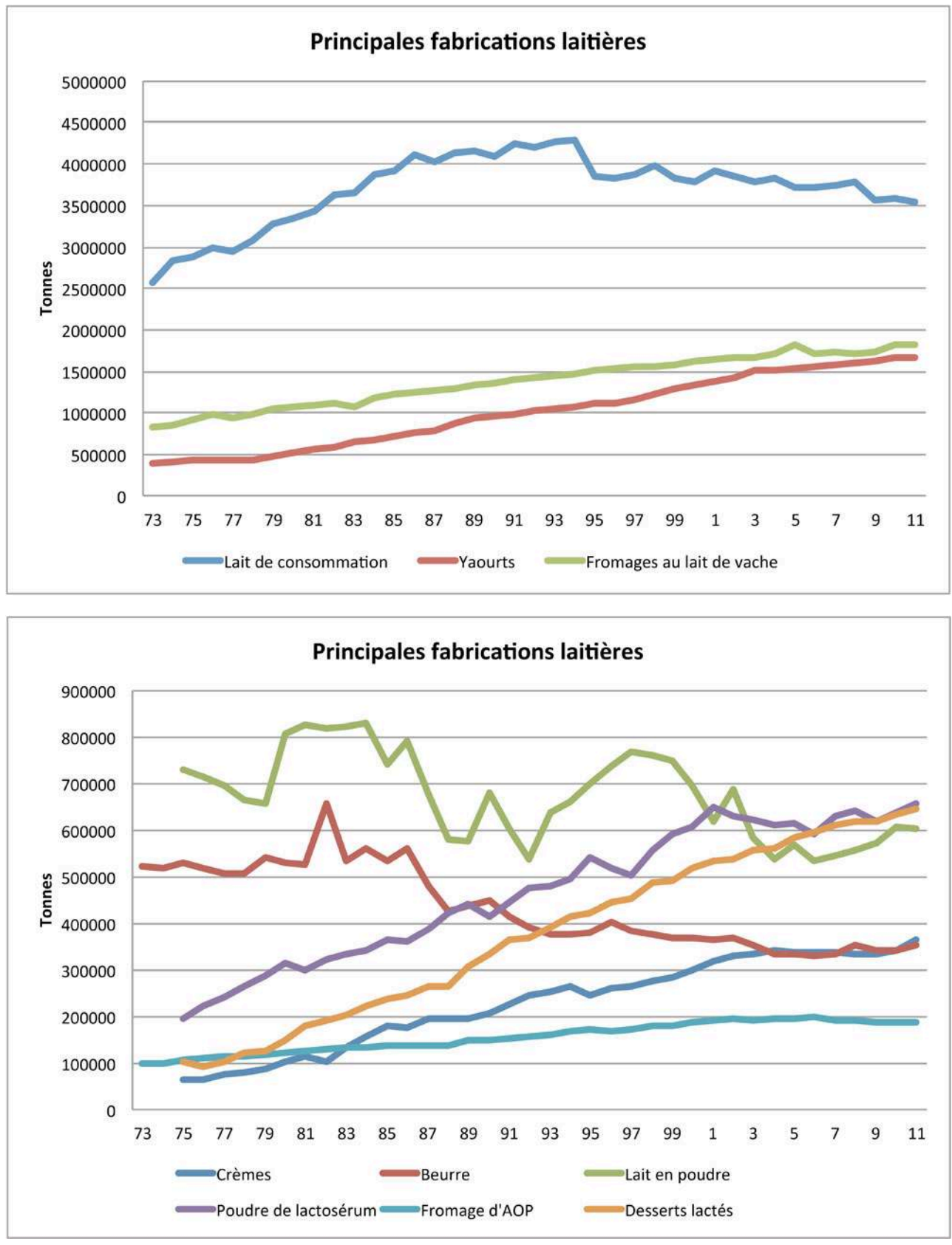

Source : CNIEL.

Beurre et poudres (de lait et de lactosérum) obéissent à des dynamiques productives spécifiques marquées par le poids du marché comme par la politique européenne. Ces fabrications avaient reculé avec la baisse de la consommation de beurre et des prix d'intervention. Le mouvement semble contenu depuis dix ans compte tenu des nouvelles opportunités de marché. Les exportations de poudre de lait écrémé hors Union Européenne sont ainsi passées de 32930 tonnes de 2009 à 108688 tonnes 2012, celles de lait infantile de $51380 \mathrm{t}$ à $80805 \mathrm{t}$ (Eurostat). Celles de poudre de lactosérum 
sont aussi très dynamiques. La France bénéficie là d'une vraie technicité et d'une image positive, même si la concurrence est rude avec les Etats-Unis, l'Europe du Nord et l'Océanie, surtout en Chine, un marché clef pour les poudres infantiles françaises.

\section{Les recompositions capitalistiques} CNIEL, 27 établissements de collecte ${ }^{27}$ sur 483 , soit 5,6\% assurent $61,7 \%$ de la collecte nationale en 2012. Mais la concentration est plus nette encore si l'on raisonne en terme d'entreprises puisque Lactalis, Danone, SODIAAL, Bongrain et Bel assurent $62 \%$ de la collecte française ${ }^{28}, 84 \%$ même si l'on prend en compte Laïta, Senoble et les rapprochements en cours Terra Lacta / Bongrain et Eurial / Agrial. Bref, huit groupes assurent l'essentiel de la transformation laitière française. La concentration est toutefois moins spectaculaire qu'en Europe du $\operatorname{Nord}^{29}$, grâce à la présence de nombreuses PME et de centaines de coopératives villageoises qui occupent des marchés

Revue Géographique de l'Est, vol. 54 / 1-2 | 2014 
de niche (fromages le plus souvent) et profitent de la bonne valorisation de certaines AOP.

Fig. 5 : L'organigramme du Groupe Bongrain

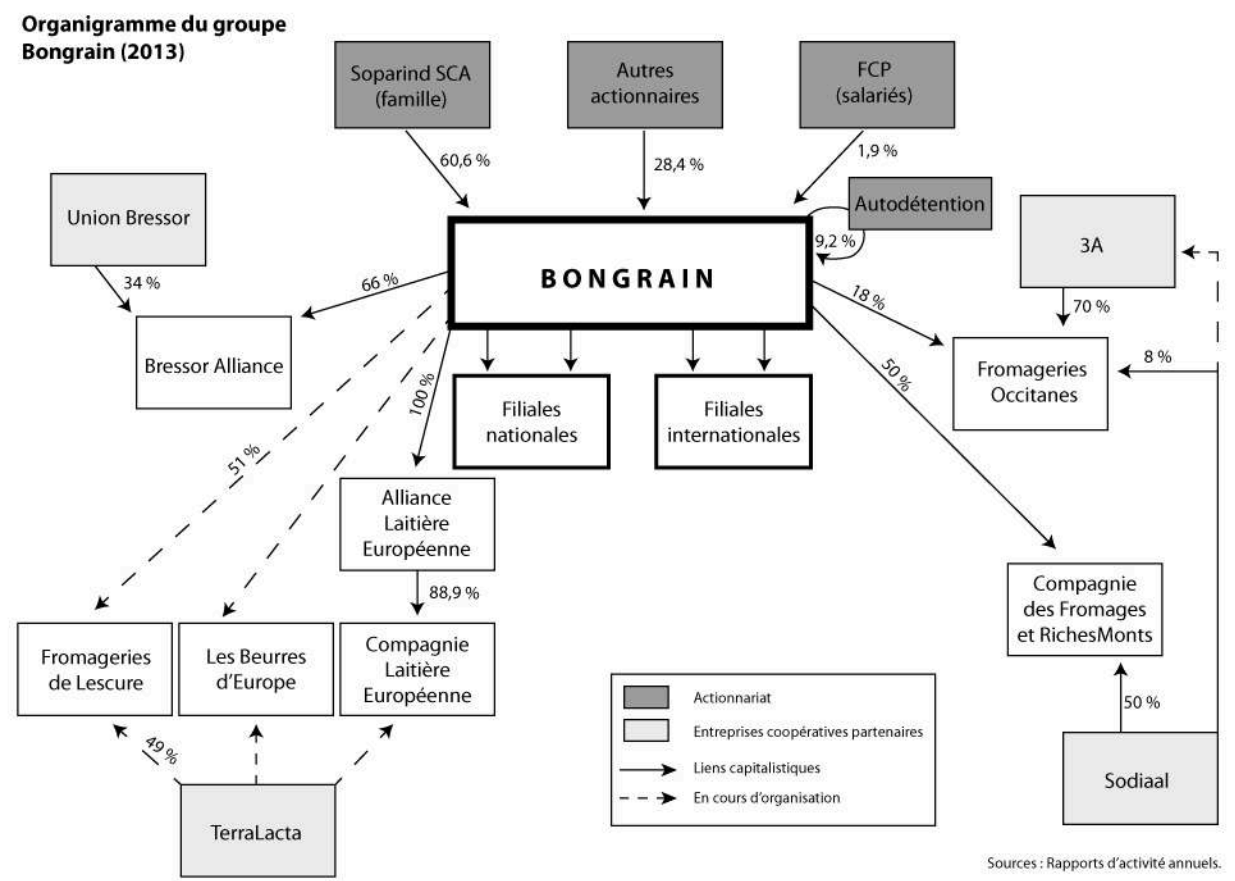

Les modalités de gouvernance de ces groupes diffèrent. La plupart ont un fonctionnement très centralisé, à l'image de Lactalis, Bel ou Danone. Dans les grosses coopératives, la prise de décision est nécessairement centralisée au niveau du siège social, mais une partie du pouvoir reste bien aux mains des producteurs (détenteurs du capital social) et des unions régionales héritées de l'histoire. Bongrain est nettement plus décentralisé, avec un organigramme complexe et de nombreux partenariats noués avec les coopératives.

Reste l'internationalisation du capital. La filière fut longtemps contrôlée par le capital national, à l'exception de quelques cas particuliers, d'une incursion avortée de l'italienne Parmalat et de la grosse usine de Dieue-sur-Meuse, rachetée par l'Allemand Hochland dès 1978. L'ouverture s'est accélérée en 2011 avec la reprise par l'Américain General Mills de $51 \%$ de Yoplait, la filiale de SODIAAL ${ }^{30}$. Elle continue aujourd'hui puisque deux groupes chinois se sont associés avec SODIAAL à Carhaix (Synutra) et la coopérative d'Isigny (Biostine) pour produire des poudres. La présence du capital étranger devient donc significative, même si on est loin des réalités en cours dans le reste de l'industrie française. A l'inverse, les grandes entreprises françaises sont massivement présentes à l'étranger, qu'il s'agisse de Senoble ou de Bel, encore plus de Lactalis, Bongrain et Danone. Elles y contrôlent certainement l'équivalent de la collecte nationale! 


\section{Les régions dans la mutation des systèmes productifs}

Les modalités de fonctionnement des filières diffèrent sensiblement selon les régions, en liaison avec le poids de l'histoire, la densité de la ressource, l'action des entreprises et, bien entendu, le rôle des hommes. L'objectif sera ici d'insister sur les mutations en cours.

\section{A. Le Grand Ouest}

Fig. 6 : La localisation du troupeau laitier dans le Grand Ouest en 2010

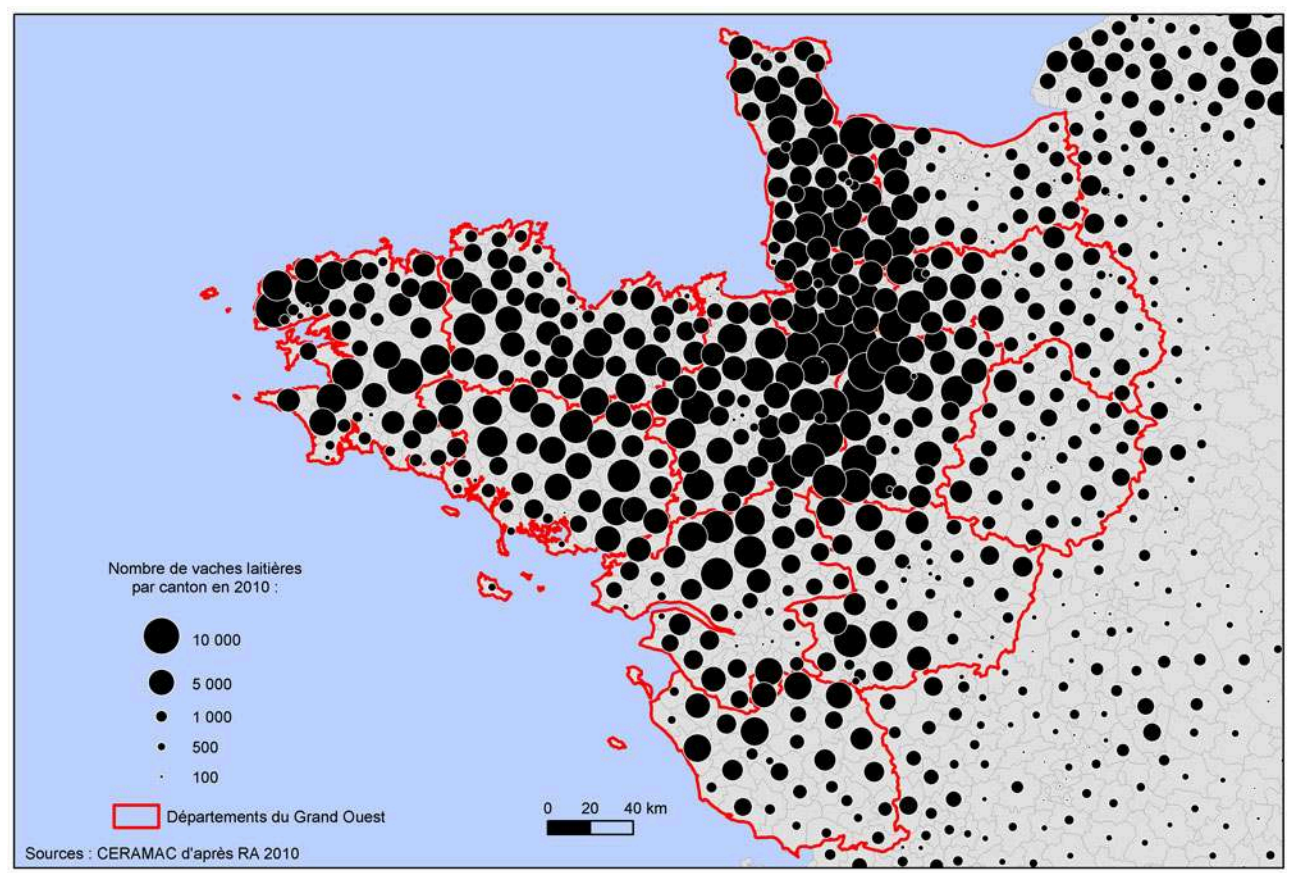

67 Le Grand Ouest s'appuie sur des dynamiques productives puissantes, bridées depuis 1984, mais dont on peut penser qu'elles vont s'exprimer à nouveau pleinement une fois les quotas abandonnés. Ces filières peuvent être décrites en quelques mots: des producteurs nombreux qui s'inscrivent dans les dynamiques intensives héritées de la révolution agricole, des bassins laitiers étendus, denses et peu coûteux à collecter, une transformation qui s'est diversifiée avec le temps et des grosses usines, contrôlées en partie depuis l'extérieur.

La carte révèle des zones laitières extrêmement denses à l'ouest de la péninsule (Léon, bassin de Châteaulin / Carhaix) ainsi que dans un vaste ensemble allant du Cotentin au Bessin, au Bocage normand, à l'est de l'Ille-et-Vilaine et à l'ouest de la Mayenne et dont l'épicentre se situerait vers Fougères. Le lait est à peine moins présent dans tout le reste de la Bretagne, le Bocage vendéen, le nord de la Loire-Atlantique, les Mauges et le Choletais, l'est de la Mayenne et l'ouest de l'Orne. Rares sont les territoires peu laitiers dans ce bloc compact : Monts d'Arrée, zones urbaines, littoral sud de la Bretagne.

On doit analyser cette filière à la lumière de la crise du modèle agricole et agroalimentaire breton révélée par les difficultés récentes des abattoirs. La profession 
semble en fait échapper en partie à cette crise. Elle apparaît, d'une part, plus compétitive que les filières avicoles et porcines qui souffrent énormément sur le grand export où elles ne sont plus compétitives. D'autre part, les incidences environnementales, bien que réelles, semblent moins problématiques que pour les élevages porcins et avicoles. Enfin et surtout, la filière dispose de plus d'atouts face à l'avenir. Ainsi, depuis les années 1980, les entreprises se sont efforcées de sortir du couple beurre + poudre pour fabriquer davantage de produits frais ou de fromages plus prisés par le marché. Et même la filière beurre + poudre bénéficie de nouvelles possibilités de valorisation, loin des produits basiques des années 1980 (beurre en cubes de $25 \mathrm{~kg}$, poudre pour l'alimentation animale...). Les investissements chinois dans la région expriment ces nouvelles opportunités. Rappelons enfin que l'élevage bénéficie de conditions climatiques favorables à l'herbe et au maïs et que les fromagers normands s'appuient sur un important patrimoine de savoir faire souligné par la présence de plusieurs AOP et surtout d'énormes usines de camembert aux marques connues : Président, Cœur de Lion, Le Rustique...

70 Mais le Grand Ouest souffre bien de certaines insuffisances, quasi structurelles, surtout face aux grands bassins laitiers d'Europe du Nord. A l'amont, les livraisons individuelles moyennes sont deux à quatre fois moins importantes que sur les rives de la mer du Nord. A l'aval, les spécialistes jugent l'outil industriel plutôt moins performant, avec des usines moins restructurées. Enfin, les entreprises régionales s'appuient sur des marques dont la notoriété reste relative : Grand Fermage, Paysan Breton, Even, Le Gall...

\section{B. Les montagnes de l'Est Central}

71 L'Est Central correspond à la zone traditionnelle des fruitières, regroupant la FrancheComté et la Savoie. La filière s'y est organisée de manière particulière pour constituer un système productif qui s'apparente à un modèle. Ce dernier, qualitatif, s'appuie sur plusieurs éléments forts : races locales, herbe, foin, refus de l'ensilage, rôle clef de la fruitière, affineurs; le tout dans un contexte marqué par une très forte dimension collective des pratiques (fruitières, syndicats fromagers très actifs...).

72 Analyser ces régions à l'aune des mutations des systèmes productifs oblige à s'interroger sur leur positionnement particulier. Elles sont orientées vers la production de fromages d'AOP ${ }^{31}$ dont on peut expliquer le succès commercial ${ }^{32}$ et économique ${ }^{33}$ par un positionnement revendiqué sur le créneau de la qualité et du haut de gamme. Ce positionnement s'appuie sur une stratégie de respect et d'affirmation du lien au terroir qui assure aux fromages une qualité spécifique recherchée par les consommateurs.

73 L'essentiel se joue en fait dans les années 1960/70 quand se généralise le modèle intensif Holstein + maïs + ensilage + industrie. Les interprofessions du comté, du reblochon et $\mathrm{du}$ beaufort s'opposent alors à la diffusion de ce modèle, préférant privilégier les races locales, le foin, les fruitières, le lait cru et le long affinage. Ce positionnement suscite des débats internes souvent vifs (refus de la Charte du Comté ${ }^{34}$ en 1970 notamment, position plus « progressiste » des syndicats agricoles...) mais il ne correspond pas à une attitude de refus du progrès. Jurassiens et savoyards souhaitent au contraire maintenir un système global privilégiant l'expression du terroir. Les précurseurs sont les acteurs du beaufort, suivis par ceux de la filière comté, qui disposent de gros volumes, de compétences humaines et d'une profession très soudée autour de sa capitale Poligny. Tous vont ensuite renforcer les cahiers des charges des 
différentes AOP à partir des années 1990 : affirmation de l'usage des races locales, du foin et du lait cru, allongements des durées d'affinage... dont elles ont su faire un vrai outil de développement, un élément clef pour ces massifs mal placés dans la concurrence interrégionale. La ressource territoriale a été bien valorisée, permettant de préserver les producteurs (qui ont moins diminué qu'ailleurs) et les outils de transformation (il reste 300 fromageries dans le Jura et en Savoie).

Il faut toutefois distinguer les vrais bassins de production (Doubs, Avant-pays savoyard) des marges moins denses (Bugey, Haut Jura, Maurienne, Chartreuse...). Il faut aussi distinguer les terres de fruitières en gestion directe ${ }^{35}$ (comté, beaufort, tome des Bauges) de celles qui pratiquent la gestion indirecte ${ }^{36}$ (Haute-Savoie). Cette dernière pratique a favorisé l'arrivée de capitaux extérieurs, les PME régionales succédant aux artisans locaux avant que Lactalis ne devienne le plus gros fruitier, sans toutefois que cette nouvelle configuration ne nuise à ce jour à l'efficacité économique de la filière.

\section{Rhône-Alpes et le Sud-Est} une partie du Forez (fourme de Montbrison) avaient une tradition fromagère ${ }^{37}$. Puis Lyon, Grenoble et Saint-Étienne se sont érigées en bassin de consommation qui ont stimulé l'organisation d'une nouvelle filière.

mutation s'engage surtout après guerre, quand certaines entreprises, notamment coopératives, prennent en charge l'approvisionnement de ces villes puis se regroupent dans l'Organisation Régionale Laitière Agricole Coopérative, l'une des unions constitutives de SODIAAL en 1990. Avec le privé, qui se structure autour de Danone et de son énorme usine de produits frais de Saint-Just Chaleyssin (banlieue lyonnaise), l'ORLAC s'inscrit dans un contexte régional marqué par la révolution agricole et l'intensification de la production. Les zones qui répondent le mieux à cette stimulation sont les plaines de l'Isère et de la Saône, mais surtout la montagne : Monts du Forez et du Lyonnais, Pilat, Est de la Haute-Loire, voire Trièves (Isère) et Valromey (Ain). Le lait se développe vigoureusement, surtout dans les années 1960/70, conduisant à une hyper spécialisation de ces moyennes montagnes avec un dense réseau de petites fermes intensives, les monts du Lyonnais représentant l'archétype de cette dynamique. 


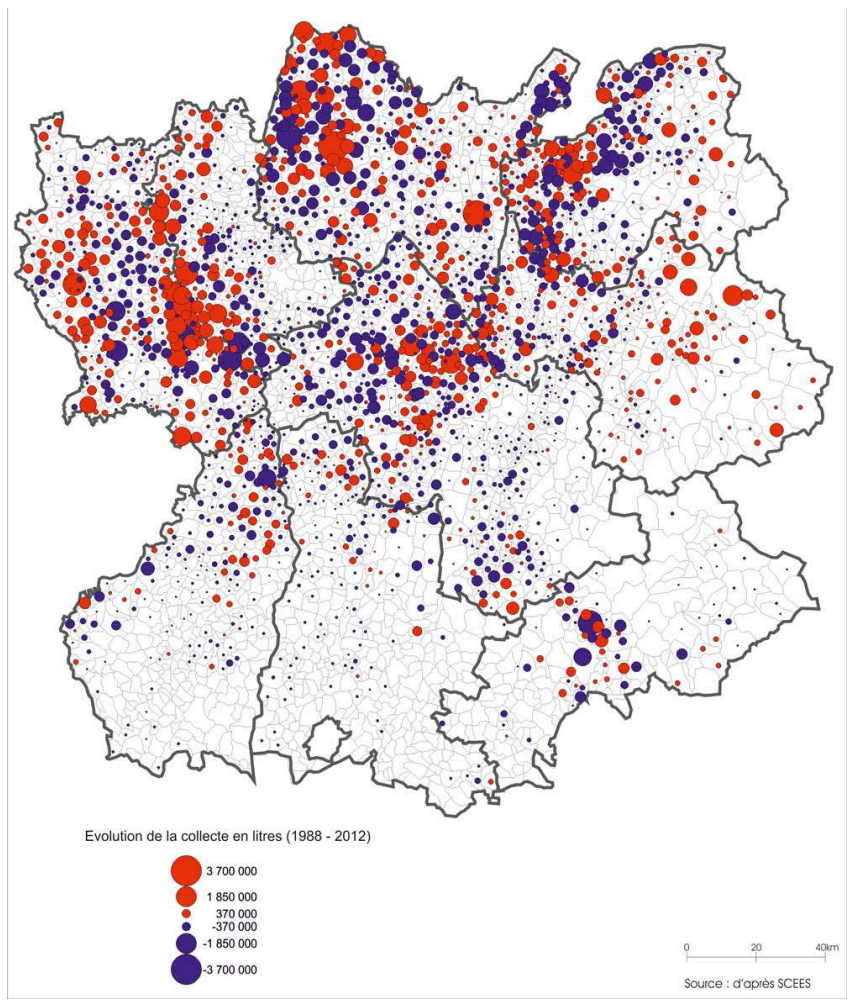

78 Les quotas (1984) bloquent la croissance des volumes, mais ne modifient pas la géographie de la production, en dehors de l'effacement du Trièves. L'intense restructuration de l'aval fait disparaître les petits artisans lyonnais et les coopératives qui ne peuvent suivre dans la course aux volumes, comme celles des Alpes du Sud. Regroupées certes dans l'URCAL ${ }^{38}$, elles sont victimes de la disparition de leur marché traditionnel de la Riviera et rejoignent l'ORLAC en 1988, étendant ainsi le bassin lyonnais jusqu'à la Durance, voire au haut Var.

Rhône-Alpes et PACA ont livré en 2012 1,489 milliard de litres, soit 6,2 \% de la collecte nationale (CNIEL), un volume inférieur à celui de la seule Ille-et-Vilaine. L'efficacité globale du système est donc relative, surtout à l'amont. L'aval s'appuie largement sur les énormes usines de Saint-Just Chaleysssin et de Vienne, qui livrent du lait Candia et des yaourts (Danone, Yoplait, marques distributrices). Réfléchir aux mutations du système productif conduit à s'interroger sur la capacité d'adaptation de ces filières.

La figure $n^{\circ} 7$ révèle les dynamiques qui animent l'amont. Les régions périphériques sont souvent en perdition, à l'image de la Drôme et des montagnes de l'Isère et des Hautes-Alpes, autant de secteurs sans AOP où le lait recule. A l'inverse, la collecte se renforce sur certains territoires ${ }^{39}$, illustration de la concentration géographique des bassins de production, en liaison avec l'existence de dynamiques productives locales et la volonté de réduire les coûts de collecte. Les vieux foyers de la révolution agricole s'affirment alors, notamment dans la Loire. En Savoie, la montagne se distingue par une croissance de la collecte qui est à relier à un traitement administratif favorable et à la dynamique spécifique des fromages d'AOP, notamment celle du beaufort. On a là une des régions laitières les plus dynamique de Rhône-Alpes, en rupture totale avec la configuration des années 1960/70. 
81 La capacité d'adaptation des systèmes productifs interroge aussi l'aval à travers de systèmes de valorisation très différenciés. La Savoie est le fief d'une transformation artisanale coopérative performante, même si le secteur privé et l'industrie y ont progressé. Autour de Lyon en revanche, la concentration industrielle associée à des productions de masse est la règle, même si l'on trouve ici et là des PME dynamiques. Restent les plaines de l'Ain, historiquement marquées par la coopération. Quelques coopératives y fabriquent du beurre et de la crème de Bresse AOP alors que celles du groupe Union Bressor (Bresse bleu) sont aujourd'hui liées à Bongrain et que celles qui adhéraient à l'URCVL ont fait naufrage.

A l'amont comme à l'aval, les enjeux régionaux renvoient au marché et à la demande des consommateurs, à l'efficacité des éleveurs et des laiteries ainsi qu'à l'évolution de la PAC.

83 En Savoie et dans le Bugey, l'orientation vers la qualité est claire et ancienne. Elle explique la permanence de la production dans ces massifs souvent difficiles où le prix du lait compense le déficit de productivité. Le Pays de Thônes et le Beaufortain sont emblématiques de cette réussite, autour de la production de reblochon fermier et de beaufort.

Les agriculteurs des autres territoires ont-ils la capacité à se projeter dans l'après quota ? En Bresse et en Dombes, ils disposent de structures performantes, à l'inverse de ceux du Pilat ou des Monts du Lyonnais. Ces massifs constituent de denses bassins laitiers, mais les agriculteurs n'y livrent que 180000 litres par an, ce qui s'accorde mal avec un système orienté vers la fabrication de produits de masse. La logique économique de ces grosses usines intègre en effet la nécessité de réduire les coûts de collecte et cela au sein d'entreprises dont les centres de décision sont désormais très éloignés de Lyon. C'est l'illustration parfaite de l'élargissement progressif du rayon d'action des filières.

Les Hautes-Alpes, elles, cumulent les handicaps: structures foncières étriquées, contrainte montagnarde, localisation périphérique, absence de valorisation locale et inclusion dans des filières ultra concurrentielles. La collecte y recule de manière structurelle, même en Champsaur. 
Fig. 8 : La transformation laitière en Rhône-Alpes

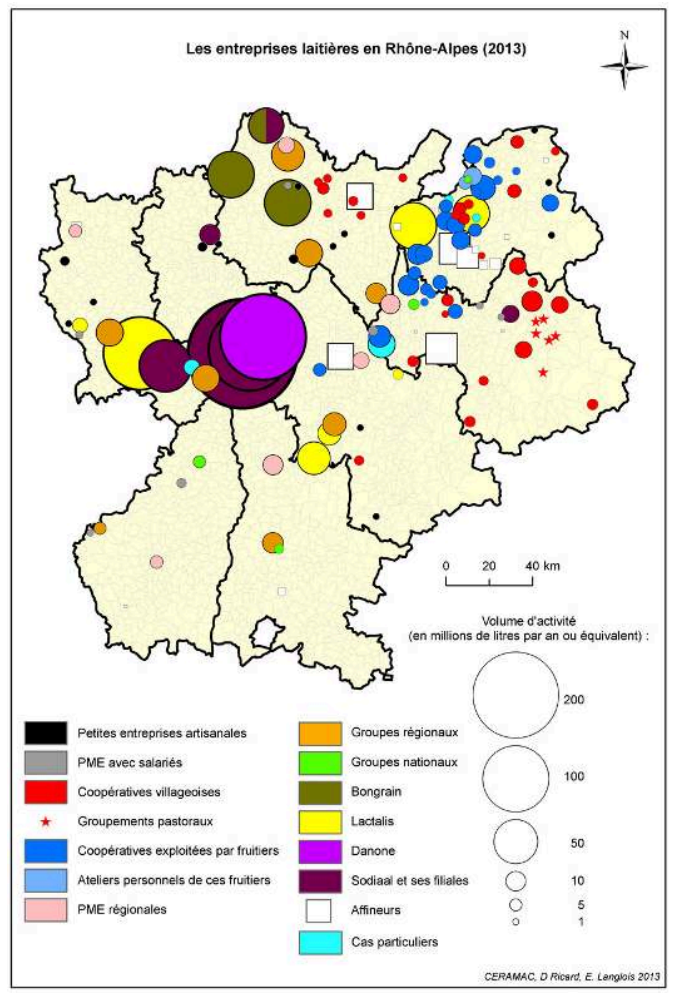

\section{Conclusion}

Réfléchir aux mutations des systèmes productifs oblige le géographe à intégrer différentes échelles d'analyse, du local au global ainsi que de multiples facteurs d'évolution, individuels ou collectifs, techniques ou commerciaux. C'est peut-être encore plus le cas dans les filières agroalimentaire où le marché n'agit pas seul, ce qui conduit à réserver une place de choix dans l'analyse à la politique agricole.

Les filières laitières sont importantes en France, présentes presque partout, à l'exception des régions méditerranéennes, voire du cœur des bassins céréaliers et elles sont fortement structurantes à travers une certaine intensité de main-d'œuvre, la collecte journalière et les liens étroits qu'elles tissent entre l'amont et l'aval et avec les territoires. Elles offrent un bon exemple de système productif globalement dynamique, pourvoyeur d'emplois et de produits transformés réputés, dynamique à l'exportation. Cette filière s'inscrit dans un environnement de plus en plus ouvert sur l'extérieur, avec une dimension européenne bien présente et une ouverture sur le grand export de plus en plus nette. Dans un tel contexte, la France souffre d'insuffisances relatives au niveau de l'amont ainsi que dans la transformation en produits de base industriels à faible valeur ajoutée, mais elle bénéficie d'autres atouts: entreprises mondialisées performantes, marques commerciales réputées, niveau technologique, produits de terroir, image positive à l'étranger... Les mutations animent continuellement la filière, tant à l'amont qu'à l'aval, selon une triple dimension technique (qui stimule la concentration des exploitations et des laiteries), productive (au niveau de l'aval, avec des évolutions différenciées selon le type de produits finis) et géographique (avec une forte incidence du facteur politique, via la PAC). 


\section{BIBLIOGRAPHY}

Canévet C., 1992, Le modèle agricole breton, PU de Rennes, 398 p.

Canévet C., 1991, 40 ans de révolution agricole en Bretagne, 1950/1990, Institut culturel de Bretagne, $294 \mathrm{p}$.

Centre National Interprofessionnel de l'Economie Laitière (CNIEL), L'Economie Laitière en Chiffres, édition annuelle de données statistiques.

Collectif, 2013, Les recompositions récentes des filières laitières en France et en Europe, CERAMAC 31, PUBP, Clermont-Ferrand, $456 \mathrm{p}$.

Delfosse Cl., 2007, La France fromagère, Paris, La boutique de l'histoire, 270 p.

Fumey G., Etcheverria O., 2004, Atlas mondial des cuisines et gastronomies, Ed. Autrement, 80 p.

Institut National de l'Origine et de la Qualité (INAO), données relatives aux AOP / IGP.

Revue Laitière Française (RLF), revue professionnelle de référence.

Ricard D., 1994, Les montagnes fromagères françaises, CERAMAC, Clermont-Ferrand, 496 p.

Ricard D., 1997, Les filières fromagères françaises, Ed. RIA, Paris 240 p.

\section{NOTES}

1. - Le beurre océanien arrive par bateaux congélateurs en Europe dès la fin du $19^{\mathrm{e}}$ siècle.

2. - CNIEL : Centre National Interprofessionnel de l'Economie Laitière.

3. - Les exploitations ayant des vaches laitières passent de 1404200 en 1963 à 728300 en 1973 (CNIEL)!

4. - La traite mécanique concerne $46,7 \%$ des exploitations en 1975 , mais $98 \%$ dès 1983 (SCEES) !

5. - Si la révolution agricole a joué son rôle, la dynamique laitière reste toutefois ici assez ancienne.

6. - La référence moyenne par exploitations est de 226295 litres en altitude contre 363295 litres en plaine (CNIEL, 2011).

7. - Aquitaine et Midi-Pyrénées livrent moins de lait que le seul département de l'Ille-et-Vilaine.

8. - Les livraisons moyennes les plus élevées se situent presque toujours dans ces plaines céréalières : 517000 litres dans la Vienne, 488000 en Indre-et-Loire, 481000 dans les DeuxSèvres, 454000 dans la Marne et l'Yonne et même 586000 litres/an pour les rares producteurs du Val d'Oise / Yvelines / Essonne (CNIEL, 2011).

9. - Leurs racines remontent toutefois souvent à l'entre-deux-guerres.

10. - SOciété de DIffusion AgroALimentaire, la plus grosse coopérative laitière nationale.

11. - Les beurreries valorisent la matière grasse issue de l'écrémage, mais on doit ensuite sécher le lait écrémé dans des tours de séchage, ce qui permet de réduire les volumes (10,6 litres de lait écrémé donnent $1 \mathrm{~kg}$ de poudre) et d'obtenir des poudres faciles à conserver.

12. - L'OCM lait repose sur un prix d'intervention auquel Bruxelles s'engage à acheter les quantités excédentaires de beurre et de poudre de lait pour les stocker et assainir ainsi le marché de ces deux produits, et donc du lait en général. L'intervention fait ainsi office de prix garanti pour toute la filière.

13. - Les produits importés font l'objet de taxes dissuasives alors que les exportations (non compétitives) bénéficient de généreuses aides à l'exportation dites restitutions. 
14. - Les stocks européens atteignent 1283000 tonnes de beurre et 771000 tonnes poudre de lait en 1986 (CEE)!

15. -Les Droits à Paiement Unique (DPU) sont des aides à l'hectare versées sans obligation de production (découplées de l'acte de production).

16. - 336 euro/tonne en 2008 , mais 274 euro l'année suivante, avec même un étiage à 210 euro au printemps.

17. - Leur rôle ne cesse de croître mais il s'agit là de produits très standardisés dont le prix est l'argument essentiel.

18. - La Holstein, au pelage noir et blanc, est la vache laitière de référence, docile et très productive, sélectionnée à la fois en Europe (Pays-Bas, Allemagne, France...) et aux Etats-Unis.

19. - La matière grasse a perdu de son intérêt avec la réduction de l'intervention sur le beurre. Rappelons que ce dernier est constitué de $82 \%$ de matière grasse.

20. - L'Institut de l'Elevage évalue le nombre de robots à 94 en 2000, 1506 en 2006 et 2785 en 2011, qui doivent certainement produire 10\% du quota national. Les gros troupeaux sont les premiers concernés, compte tenu de l'ampleur de l'investissement (autour de 150000 euro).

21. - Soit la référence moyenne de 1995. Cette donnée inclut les livreurs directs (producteurs fermiers) dont les quotas sont surreprésentés dans cette classe statistique (FranceAgriMer).

22. - La microfiltration est un procédé non chauffant (à la différence de la pasteurisation $\left(72^{\circ}\right)$, et encore plus de l'UHT), donc moins agressif et non destructeur pour la flore lactique. Ce type de lait (lait Marguerite...) se conserve 14 jours avant ouverture contre 7 jours pour le lait pasteurisé traditionnel.

23. - Le secteur spécifique des AOP est aussi en croissance, mais avec un rythme de progression moins rapide. Sur ce marché beaucoup plus mature, la croissance des volumes (108 809 t en 1975, $187539 \mathrm{t}$ en 2011) doit beaucoup à la multiplication des appellations. Le fromage de comté est incontestablement le plus dynamique.

24. - UNICOPA s'était rapproché d'Entremont peu avant.

25. - URCVL : Union Régionale des Coopératives de Vente de Lait.

26. - Senoble, un des leaders français des produits frais, est très implanté dans des zones laitières en déclin.

27. - Il s'agit des établissements ayant une collecte propre, une entreprise pouvant avoir plusieurs établissements.

28. - Estimations personnelles, l'évaluation des volumes collectés ou transformés étant très difficile.

29. - Il ne reste guère ici qu'une énorme coopérative par pays : Valio en Finlande, DMK en Allemagne du Nord, Friesland Campina aux Pays-Bas et Arla au Danemark et en Suède.

30. - SODIAAL avait du céder, en $2002,50 \%$ de sa filiale au fonds Paribas Affaires Industrielles. Une fois l'entreprise redressée, PAI revend sa participation, sans que SODIAAL n'ait les moyens de la racheter.

31. - Il y a 4 AOP dans le Jura (mont d'or, comté, morbier, bleu de Gex) et 5 en Savoie (abondance, beaufort, reblochon, tome des Bauges, chevrotin), plus les IGP gruyère, tomme de Savoie et emmental grand cru.

32. - La production de comté est passée de 35000 tonnes dans les années 1980 à 50000 tonnes aujourd'hui, celle de beaufort de moins 1000 tonnes en 1970/75 à 5000 tonnes (INAO).

33. - Le prix du lait oscille entre 320 et 350 euro / tonne en France avec de faibles écarts entre les régions et les entreprises. Mais on atteint 450 euro en comté, 450 à 500 euro en Haute-Savoie et on dépasse 600 euro en beaufort, tandis que les fromages d'AOP auvergnats ou normands ne dégagent presque aucune plus value par rapport au lait standard. Il y a donc bien une spécificité de l'Est Central.

34. - Impulsée par l'Etat, elle visait notamment à regrouper les fruitières au profit d'ateliers industriels plus productifs (Ricard, 1994). 
35. - La fruitière salarie du personnel et vend ses productions fromagères afin de payer le lait de ses adhérents.

36. - La fruitière possède un atelier mais vend sa collecte à un artisan, le fruitier, qui travaille dans le local de la coopérative.

37. - Plus au Sud, le Queyras se couvrit d'un dense tissu de fruitières dès les années 1840.

38. - URCAL : Union Régionale des Coopératives Agricoles Laitières.

39. - 2276 communes de Rhône-Alpes et de PACA étaient collectées en 1988 contre seulement 1581 en 2012. La part des communes livrant plus d'un million de litres passe de 59,4\% de la collecte totale en 1988 à 70,2 \% en 2012. Celles de plus de 2 millions de litres progressent de 29 à $38,1 \%$. Quant aux communes livrant plus de 5 millions de litres, leur nombre passe de 6 à 20 et leur part de 3 à 9,5\% (RGA 1988, CNIEL) !

\section{ABSTRACTS}

The article deals with contemporary mutations of bovine dairy channels in France, classically associating upstream activities (producers) and downstream activities (transformers), the whole thing being in very strong interaction with the market. Those channels undergo considerable mutations linked to general dynamics of economy (competitivity demand, economies of scale, mechanization...) together with trade specificities (evolution of breeding methods, technological innovation in dairy industry, role of world markets...). Furthermore, this channel is strongly influenced by the Common Agricultural Policy (CMO milk, quotas, guaranteed prices, public stocking...). The article questions the geography of production and its evolution, as well as the mechanism of producers' restructuration. Then it insists on the downstream aspect, with a privileged approach of companies (what are they? What implantations? What productions? What capitalistic recompositions? Regional analyses of the last part combine mutations of upstream and downstream aspects in the Great west, the Jura and Savoy and in Rhône-Alps, with a certain number original maps.

L'article s'intéresse aux mutations contemporaines des filières laitières bovines en France, qui associent, classiquement, un amont (les producteurs) et un aval (les transformateurs), le tout en interaction très forte avec le marché. Ces filières font l'objet de considérables mutations liées à la dynamique générale de l'économie (exigence de compétitivité, économies d'échelle, mécanisation...) ainsi qu'aux spécificités de la profession (évolution des méthodes d'élevage, innovation technologique en laiterie, rôle des marchés mondiaux...). De plus, cette filière est fortement influencée par la Politique Agricole Commune (OCM lait, quotas, prix garantis, stockage public...). L'article questionne la géographie de la production et son évolution, ainsi que le mécanisme de restructuration des producteurs. Il insiste ensuite sur l'aval, avec une approche privilégiée par les entreprises (Quelles sont-elles? Quelles implantations? Quelles productions? Quelles recompositions capitalistiques ?). Les analyses régionales de la dernière partie combinent les mutations de l'amont et de l'aval dans le Grand Ouest, le Jura et la Savoie et en Rhône-Alpes, avec un certain nombre de cartes inédites. 
INDEX

Mots-clés: filières, France, lait, mutations, systèmes productifs

Keywords: food chains, France, milk, mutations, production systems

\section{AUTHOR}

\section{DANIEL RICARD}

Clermont Université, Université Blaise Pascal, EA 997 CERAMAC, BP 10448, F-63000 ClermontFerrand, France 